\title{
Diepes, Christoph (2018): Klimaschutz und Klimaanpassung in der verbindlichen Bauleitplanung. Eine vergleichende Analyse ausgewählter Städte
}

\author{
Lemgo: Verlag Dorothea Rohn. 15 Tabellen, 22 Abbildungen, 2 Anhänge, 212 Seiten
}

\section{Ulrike Weiland $^{1}$}

Online publiziert: 3. Juli 2018

๑) Springer-Verlag GmbH Deutschland, ein Teil von Springer Nature 2018

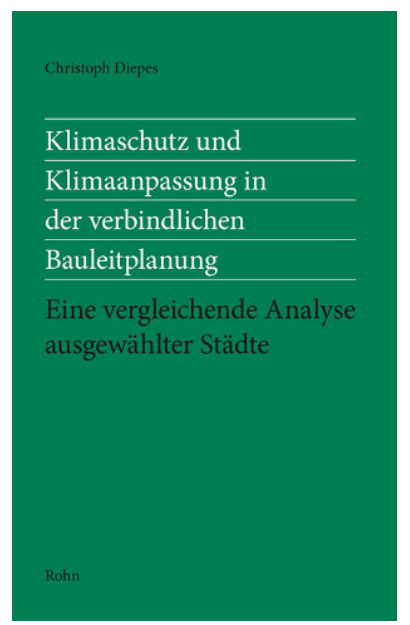

Der Autor greift mit seiner Arbeit ein aktuelles, relevantes und interessantes Thema auf: Wie reagieren Städte mit dem Instrument der Bebauungsplanung auf den Klimawandel? Literatur zu kommunalen Handlungsmöglichkeiten beim Klimaschutz und zur Anpassung an den Klimawandel gibt es inzwischen viel, auch viele Auflistungen guter (Umsetzungs-)Beispiele, aber eine empirische Untersuchung, wie Klimaschutz und Anpassung an den Klimawandel in der verbindlichen Bauleitplanung aufgegriffen werden, ist neu.

Das Thema ist komplex, da sowohl Stadtklima und Klimawandel als auch Städte und ihre Planung und Entwicklung miteinander verbundene - gekoppelte - komplexe Systeme darstellen, deren Steuerung alles andere als trivial ist.

Prof. Dr. Ulrike Weiland

uweiland@uni-leipzig.de

1 Institut für Geographie, Universität Leipzig, Johannisallee 19A, 04103 Leipzig, Deutschland
Dazu gehört, dass Städte im Klimawandel eine ambivalente Rolle einnehmen, nämlich die der Hauptverursacher des anthropogenen Anteils und die der besonders Betroffenen von den Auswirkungen des Klimawandels. Dementsprechend viele Facetten der Thematik sind abzubilden, was dem Autor sehr gut gelungen ist.

Aufgrund der kenntnisreichen Darstellung sowohl kommunaler Handlungsmöglichkeiten als auch der Praxis ausgewählter Kommunen, Klimaschutz und Anpassung an den Klimawandel vor allem mit der verbindlichen Bauleitplanung umzusetzen, wird das Buch eine ergiebige Informationsquelle für alle, (1) die sich in der Planungspraxis mit Klimaschutz, Anpassung an den Klimawandel bzw. klimawandelgerechter Stadtentwicklung befassen, (2) die sich im Studium darauf vorbereiten möchten und/oder (3) die sich über die Klimapolitik der drei untersuchten Städte informieren wollen. Je nach Interessenschwerpunkt kann das Buch selektiv gelesen werden.

Der Autor hat knapp 1.000 Bebauungspläne der Städte Frankfurt am Main, Stuttgart und München aus den Jahren 1990 bis 2013 daraufhin untersucht, inwieweit sie klima(wandel)relevante Themen aufgreifen und Maßnahmen zum Klimaschutz und zur Anpassung an den Klimawandel vorsehen. Ziel des Buches ist, zu einer „Erhöhung des Kenntnisstandes zur praktizierten Verwendung klimarelevanter Maßnahmen in der verbindlichen Bauleitplanung“ beizutragen (S. 3); eine Vollzugskontrolle der Umsetzung der Bebauungspläne ist nicht intendiert. Da die Anzahl der untersuchten Pläne fast einer Vollerhebung in den drei Städten entspricht, kann die Untersuchung als repräsentativ angesehen werden. Die Städte wurden ausgewählt, da sie als Vorreiter kommunaler Klimapolitik gelten und wegen ihrer wirtschaftlichen Prosperität auch über die Ressourcen für eine anspruchsvolle Klimapolitik verfügen. 
Die der eigentlichen Untersuchung in Kapitel 2 vorangestellten Grundlagen und Begriffe zum Klimawandel sind als Hinführung zum Thema sehr hilfreich und stellen aufgrund ihrer Beschränkung auf wesentliche Inhalte eine sehr gut lesbare Einführung zu den Herausforderungen des Klimawandels für Städte dar. Kapitel 3 ist ein nützliches Kompendium der Vorgaben und Instrumente der Stadtplanung in Deutschland für den Umgang mit dem Klimawandel, angefangen von verfassungsrechtlichen Rahmenbedingungen über das Energiefachrecht und seinem Verhältnis zur Stadt- und insbesondere Bauleitplanung bis hin zu Handlungsmöglichkeiten und Verpflichtungen zu Klimaschutz und Anpassung in der Bauleitplanung - wobei zu berücksichtigen ist, dass die Analyse des Baugesetzbuchs mit dem Jahr 2013 endet.

Für die empirische Untersuchung der Bebauungspläne und deren Begründungen mit darin enthaltenen Umweltberichten entwickelt der Autor in Kapitel 4 einen Untersuchungsrahmen mit sechs Haupthandlungsfeldern und 18 Unterkategorien bzw. Handlungsfeldern, der sich bewusst vom Baugesetzbuch löst. Das Erkenntnisinteresse ist auf die Gewinnung ,klimarelevanter Resultate ' ausgerichtet, die durch verschiedene Vorgaben des Baugesetzbuches, durch explizit oder nur implizit klima(wandel)bezogene Maßnahmen und durch das Fachrecht begründet werden können. In den Haupthandlungsfeldern Energie, Bebauungsstruktur und Gebäudekonfiguration, großflächige Grünstrukturen und Freiflächen, kleinteilige Grünstrukturen, Wasserhaushalt und Verkehr werden die Bebauungspläne jeweils anhand mehrerer Unterkategorien untersucht (S. 80 ff.). Als Erhebungsmethoden setzt der Autor Dokumentenanalysen und Experteninterviews ein.

Kapitel 5 ist vor allem für diejenigen lesenswert, die sich für die Klimapolitik der drei untersuchten Städte interessieren, denn darin werden als Kontext für die folgende Auswertung der Bebauungspläne die Klimaaktivitäten von Frankfurt am Main, München und Stuttgart, deren Historie und Instrumente sowie der jeweilige Stellenwert der Bauleitplanung skizziert. In allen drei Städten gibt es zahlreiche programmatische Grundlagen für Klimaschutz und Anpassung an den Klimawandel, die in dem Buch gar nicht im Detail dargestellt werden können. Es wird klar, dass Frankfurt deutlich mehr auf kooperative Lösungen als auf Bebauungspläne setzt als die beiden anderen Städte. ${ }^{1}$

In der Analyse und Evaluation der Bebauungspläne in Kapitel 6 kontrastiert der Autor seine empirischen Ergebnisse mit programmatischen Aussagen der betreffenden Städte zu Klimaschutz und Anpassung an den Klimawan-

\footnotetext{
${ }^{1}$ Untersuchte Bebauungspläne mit klimarelevanten Inhalten in Frankfurt am Main: 135, München: 397, Stuttgart: 419. Dies entspricht laut Autor jeweils über 90 Prozent der ab 1990 bis zum ersten Quartal 2013 aufgestellten Pläne.
}

del. In der Untersuchung der Häufigkeit des Einsatzes klimarelevanter Handlungsfelder, die anhand des beschriebenen Untersuchungsrahmens durchgeführt wird, werden auch Spitzenwerte und Nachholbedarf benannt. Dabei zeigt sich ein sehr heterogenes Bild: In allen drei Städten werden zwar häufig klimarelevante Aussagen zu Dachbegrünungen, Bepflanzungen und zum ruhenden Verkehr gemacht und übereinstimmend besteht Nachholbedarf bei der Förderung des ÖPNV. Die übrigen Handlungsfelder werden von den Städten jedoch unterschiedlich gewichtet, sodass beispielsweise in den Handlungsfeldern Energie, Bebauungsstruktur und Gebäudekonfiguration oder Wasserhaushalt große Unterschiede in der Häufigkeit der vorgesehenen Maßnahmen bestehen (vgl. Tabelle 14, S. 140). Im Ergebnis fließen die Aufgaben, die sich mit dem Klimawandel stellen, ,nur sehr zaghaft [...] in die verbindliche Bauleitplanung" ein (S. 152), und das, obwohl in den drei untersuchten Städten zahlreiche klima(wandel)relevante kommunale Institutionen, Programme und Daten vorhanden sind!

Schlussendlich stellt der Autor eine Diskrepanz zwischen Absichtsbekundungen und Handlungsmöglichkeiten der Städte einerseits und der tatsächlichen Implementation klima(wandel)relevanter Regelungen in der Bebauungsplanung andererseits fest. Die möglichen Gründe hierfür seien vielfältig und reichten von Maßstabs- und Genauigkeitsunterschieden zwischen Klimamodellen und Planungsaufgaben, dem Fehlen konkreter Grenz- und Schwellenwerte und Zielvorgaben für die Klimaanpassung über Mehrkosten, die Priorisierung der Wirtschafts- und Standortpolitik gegenüber der Umwelt- und Klimapolitik, bis zur fehlenden Innovationsbereitschaft in der Bauleitplanung und zur mangelnden Abwägungsfestigkeit von Klimabelangen wegen der überwiegenden Freiwilligkeit der Aufgabe Klimaschutz und Anpassung.

Als Handlungsempfehlung plädiert der Autor in Kapitel 7 für die Einführung einer Zwischenebene zwischen kommunalen Klimaschutzkonzepten und stadtweiten Handlungsprogrammen einerseits und der Bebauungsplanung andererseits, für die Entwicklung stadtweiter Standards und für teilräumliche Konkretisierungen der Klimabelange durch gebietsscharfe Konzepte, die wie eine Masterplanung der Bebauungsplanung vorgeschaltet sind. Ob damit alle oben genannten Problemursachen angemessen angegangen werden, darf bezweifelt werden.

Der Umgang mit Klimawandelfolgen ist in den letzten Jahren zu einem relevanten Handlungsbereich kommunaler Umweltpolitik geworden, was sich jedoch offensichtlich (noch?) nicht in gleicher Intensität in der Bebauungsplanung niederschlägt. Zwischen übergeordneten klimarelevanten kommunalen Konzepten und der Bebauungsplanung besteht - zumindest in den drei untersuchten Städten - ein Implementationsdefizit, wie es auch für andere Politikfelder nachgewiesen wurde. Wenn dies jedoch schon in den drei 
untersuchten Vorreiterstädten so ist, wie sieht es dann in anderen Städten mit geringerer Ressourcenausstattung oder in kleineren Städten aus?

Dem Autor ist zugute zu halten, dass er an mehreren Stellen anspricht, dass für Klimaschutz und Anpassung an den Klimawandel nicht nur die Bebauungsplanung, sondern auch Instrumente aus anderen Rechtsgebieten und kooperative Instrumente wie städtebauliche Verträge relevant sind, wenngleich er den Stellenwert der Bebauungsplanung für Klimabelange im Verhältnis zu den anderen Instrumenten nicht analysiert - was vermutlich den Rahmen der vorliegenden Arbeit gesprengt hätte. So verdienstvoll und nützlich das Buch ist - die These des Autors, dass ,die räumliche Planung die Grundlage und das erste Glied bei der Reduzierung von Treibhausgasen und der Vermeidung von Klimarisiken“ bilde (S. 30), muss rela- tiviert werden angesichts der Bedeutung (inter)nationaler Umweltschutz- und insbesondere Klimaschutzpolitik, des Immissionsschutzes und der betrieblichen Bestrebungen zu Ressourceneinsparungen, die ihrerseits Voraussetzungen für die räumliche Planung beinhalten bzw. die Höhe von Treibhausgasemissionen beeinflussen. Bedauerlich, aber dem Redaktionsschluss geschuldet, ist die Tatsache, dass die BauGB-Novellen 2014 bis 2017 nicht berücksichtigt werden konnten. Damit hat die Darstellung der baurechtlichen Grundlagen zu Klimaschutz und Anpassung an den Klimawandel für die jetzige Zeit nur eingeschränkt Gültigkeit. Die übrigen Aussagen, das festgestellte Implementationsdefizit und die Handlungsempfehlungen des Autors treffen jedoch nach wie vor zu und bleiben relevant, sodass das Buch unter dieser Prämisse für die genannten Zielgruppen sehr lesens- und empfehlenswert ist. 